\title{
The Informatics of the Planning Target Volume - why it cannot and should not be changed
}

Editorial

\author{
Alexis A. Miller ${ }^{1,2} *$ Scott Turner ${ }^{2 \dagger}$ \\ 1 Centre for Oncology Informatics, Faculty of Engineering \& Information Science, University of Wollongong, Wollongong \\ NSW, Australia \\ 2 Department of Radiation Oncology, Illawarra Cancer Care Centre, Wollongong NSW, Australia
}

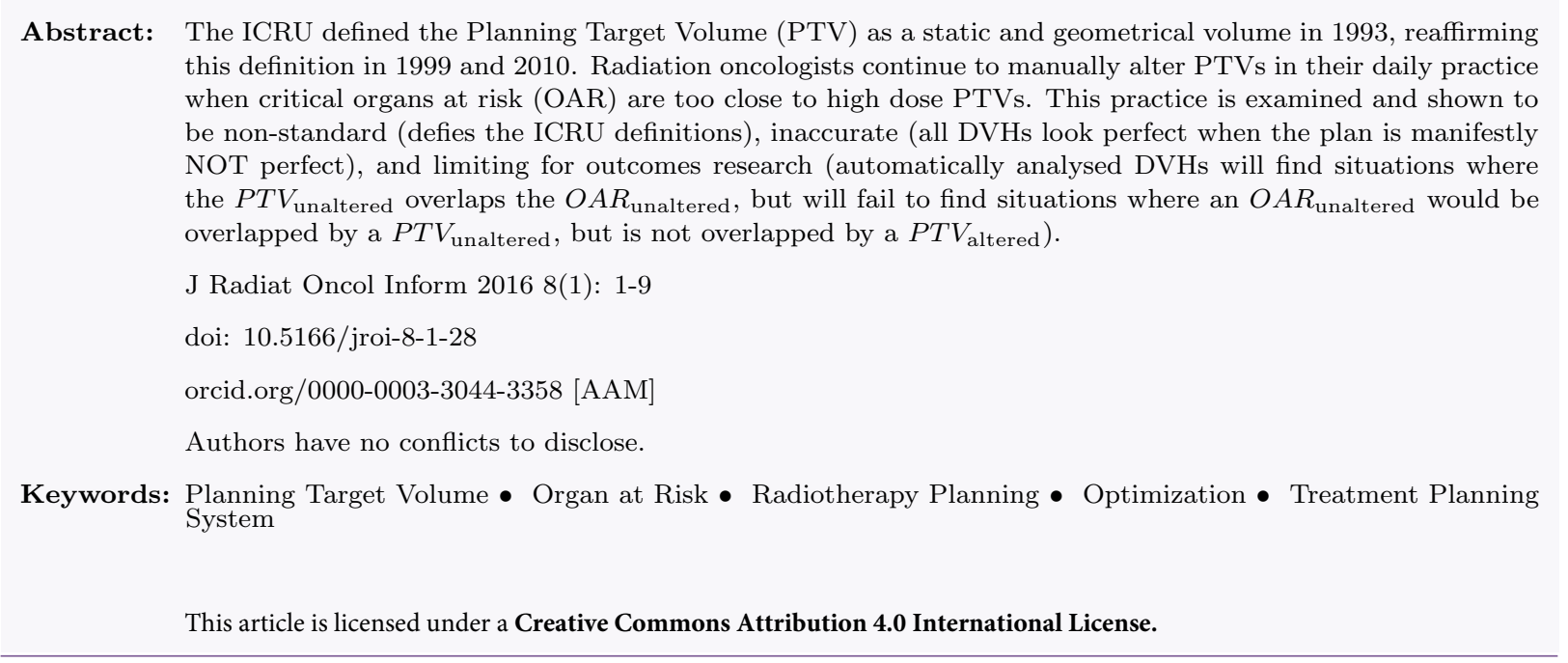

\section{Introduction}

In a recent $\mathrm{ASTRO}^{1}$ Spring Refresher course, panellists treating H\&N, lung and GIT cancers were asked if they altered their Planning Target Volumes (PTVs). It was surprising to hear all the panellists replied "yes".

The practice of altering the PTV therefore appears to be common but variable (hence the question for clarification). The usual reason is so that planners can sculpt dose away from a critical OAR, for which a maximum dose is specified, where there is overlap with a PTV requiring a higher dose. However it is not clear that this is the only or proper way to handle this occurrence. In particular this practice raises some questions -

* E-mail: alexisandrew@gmail.com,m: +61406654239

† E-mail: scott.turner@sesiahs.health.nsw.gov,au,p: +6124222 5200

1 American Society for Radiation Oncology 
1. Is this 'permitted', i.e, does it match ICRU definitions?

2. Is this truthful, i.e., does it accurately convey the clinical decision making inherent in plan construction?

3. Is this helpful, i.e., does it allow automated analysis for outcomes and quality research, in particular, is it helpful for Informatics research?

It is the assertion of this paper that the practice of PTV alteration is unhelpful and does not progress the profession in its quest to improve patient outcomes by systematically altering information.

\subsection{Is it permitted?}

As described above, it is obvious that this practice occurs and in all probability is being taught to trainees. Yet professional groups in radiation oncology, such as the ICRU, has attempted through various reports to ensure that "clear, well defined and unambiguous concepts and parameters are used for reporting purposes to ensure a common language between different centers" [2]. The far-sighted ICRU reports on Prescribing, Recording and Reporting Photon Beam Therapy from 1993 (ICRU-50 [2]) and the additional supplement from 1999 (ICRU-62 [3]) were constructed for the express purpose of standardising nomenclature and concepts in the planning process. In modern IT speak, the ICRU has tried to make us "interoperable". Both documents contain a definition of the PTV which state:

The Planning Target Volume (PTV) is a geometrical construct, and it is defined to select appropriate beam sizes and beam arrangements, taking into consideration the net effect of all the possible geometrical variations and inaccuracies in order to ensure that the prescribed dose is actually absorbed in the $C T V$.

The Planning Target Volume is thus a static, geometrical concept, used for treatment planning and for specification of dose. Its size and shape depend primarily on the CTV, but also on treatment technique used.

p. V, ICRU-50, $1993[2]$

and,

different factors to be taken into account when delineating the Planning Target Volume (PTV) and the corresponding margins deserve more accurate identification ...

Segregating the Internal Margin and the Set-up Margin reflects the differences in the source of uncertainties...

The global concept and definition of the PTV as given in ICRU Report 50 is not changed.

p. viii, ICRU-62, 1999 [3]

The most recent ICRU Report 83 [1] from 2010 has re-iterated, extended and refined these issues in the setting of Intensity Modulated Radiation Therapy (IMRT). The older definitions have become increasingly clear. 
In earlier ICRU documents, the possibility of compromising the margins of the PTV if they encroached on $O A R$ was suggested (ICRU, 1999; 2004; 2007), but is no longer recommended (my emphasis). p. 46, ICRU-83, $2010[1]$

and along with a graphical representation (Figure 4.7, p48 [1] it recommends,

To ensure accurate reporting of absorbed dose to the PTV in cases for which the PTV encroaches or overlaps another PTV, OAR, or PRV, it is now recommended that the delineation of the primary PTV margins should not be compromised. [see Figure p. 47, ICRU-83, 2010 [1]

From an artistic viewpoint, the ability of a radiation oncologist to accurately expand a CTV in 3D while looking at a 2D CT slice is suspect. Treatment Planning Systems (TPS) have the functionality to expand a CTV in 3D, either symmetrically or asymmetrically. However the TPSs also permit a PTV to be manually constructed, and if constructed according to ICRU guidelines, to be manually altered.

While manual PTV alteration can be done, ought it be done? The vendors of Treatment Planning Systems could make the PTV immune to alteration, but given the current variability of adherence to ICRU, keeping the customer happy gets a higher priority.

Similarly, our electronic system permits the treatment of a patient with high dose radiotherapy without a diagnosis, but ought we do this? The training of each new radiation oncologist lays heavy emphasis on knowing the diagnosis before acting, yet our information systems permit treatment without an entered diagnosis. This discrepancy results from a desire not to have to enter a diagnosis, rather than a desire to treat the undiagnosed.

The notion of manual construction of a PTV, or manual alteration of a generated PTV is clearly absent from the definition, unless one's name is Dr Geometry! Thus while a radiation oncologist is able alter a PTV, this is in contravention of the defined conventions of PTV construction espoused by the ICRU as the altered volume becomes neither static, nor geometrical.

In actuality, alteration of the ICRU PTV is akin to only drawing half an OAR to make a DVH look acceptable.

\subsection{Is it truthful?}

Accuracy, verifiability and quality assurance are hallmarks of radiation oncology planning practice. To deviate from these ideals is to reduce the truth of our radiation plans. The example that follows serves to illustrate why the altered PTV is not truthful, in the sense that it creates the false impression of perfection where substantial compromises exist. 
The example uses a brain tumour where the risk volume resides beside the brainstem (Figure 1a). The target radiation dose to maximize control in the PTV is defined as 6000cGy and the tolerance of the BRAINSTEM as 4500cGy. The Organ at Risk (OAR) and the Gross Tumour Volume (GTVp) and Clinical Target Volume (CTVp) are defined (Figure 1b), and the CTVp and OAR are in close proximity.

Both volumes are expanded to accommodate motion $(0.4 \mathrm{~cm}$ in the head using orthogonal port films $)$ and produce a Planning Risk Volume (BRAINSTEM_PRV_4500) and Planning Target Volume (PTV6000). The PRV and PTV are overlapping (Figure 2).

At the planning process, one of two acceptable dose distributions could be produced:

1. A plan resulting from giving the BRAINSTEM_PRV_4500 a higher priority than the PTV6000. The dose homogeneity throughout the PTV is poor, but the BRAINSTEM was not 'overdosed'. This plan would be expected to deliver minimal control and minimal toxicity. The accompanying DVH (Figure 3, dotted lines) shows clearly that this plan is not perfect (cold PTV).

2. A plan resulting from giving the PTV6000 a higher priority than the BRAINSTEM_PRV_4500. The dose homogeneity throughout the PTV is excellent, but the BRAINSTEM receives total dose. This plan would be expected to deliver maximal control and maximal toxicity. The accompanying DVH (Figure 3, solid lines) shows clearly that this plan is not perfect (hot BRAINSTEM).

It is important to understand that the two solutions represent a clinical compromise and are not perfect. The chosen solution will reflect a clinical decision regarding the rates and consequences of failure to control disease and development of side effects. Inaccurate estimates of either will sway clinical decision making. Unfortunately there is no data to help a clinician to rationally select the PTV or PRV as the primary structure. It is unlikely

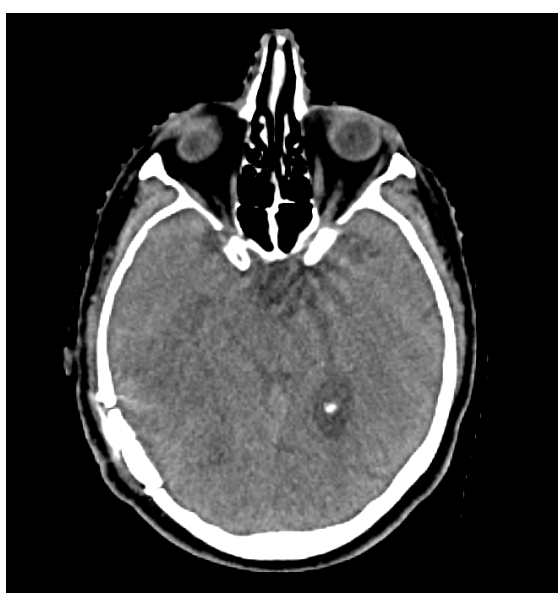

Figure 1a The CT scan showing an ill-defined brain tumour in the right parieto-occipital area under the bone flap.

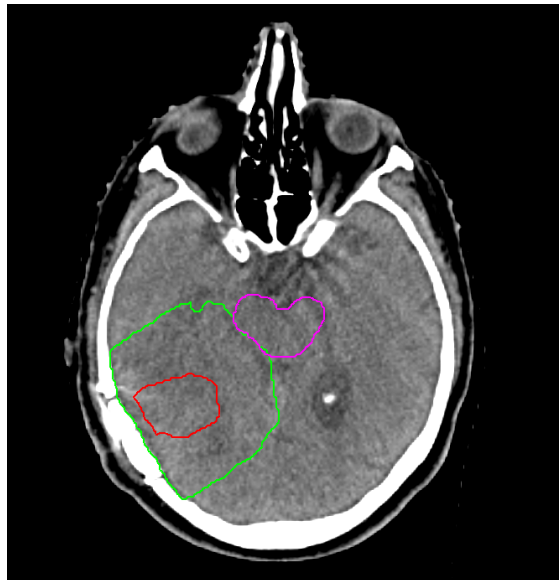

Figure 1b The CT scan showing the BRAINSTEM (purple) beside the CTVp (green) which encases the GTVp (red). 


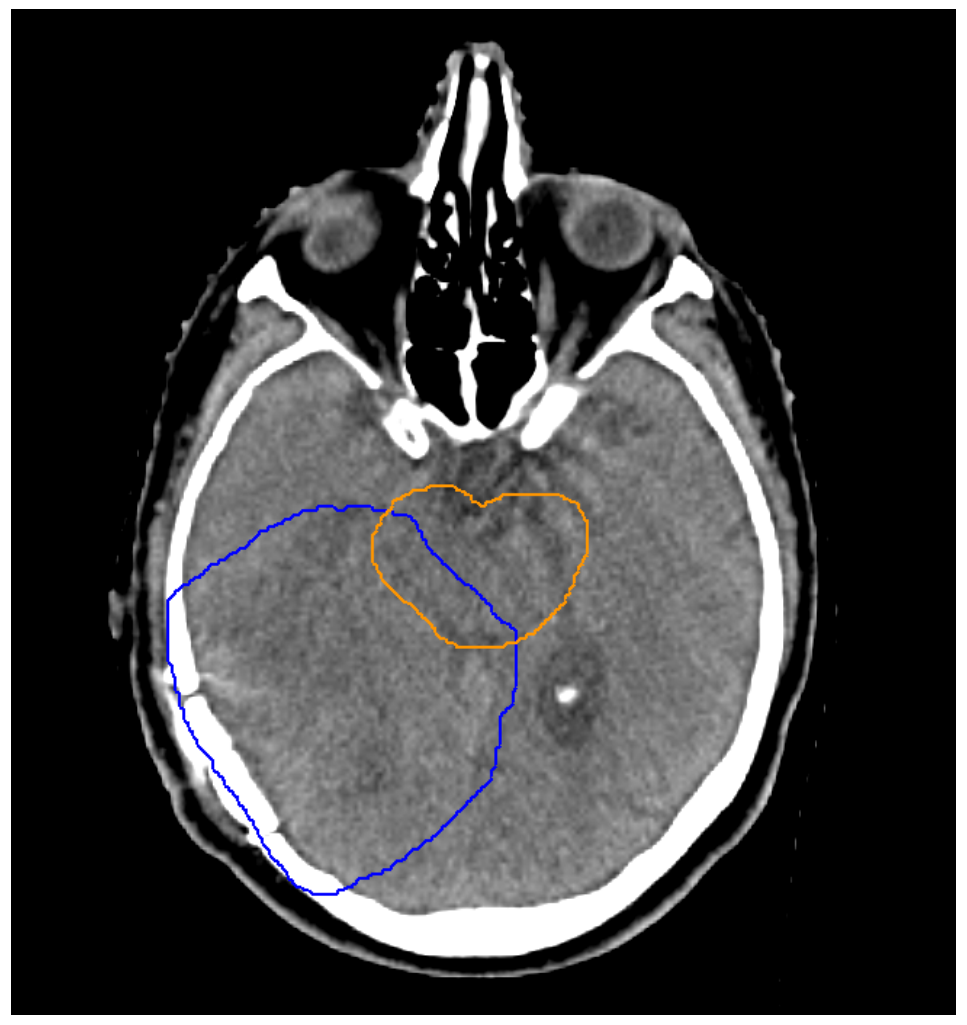

Figure 2: The CT scan showing the overlapping BRAINSTEM_PRV_4500 and PTV6000.

that any one clinician will have sufficient cases to be able to establish the preference.

So far it has been shown that, without altering the OAR or PTV, it is possible to honour the PTV dose or honour the OAR tolerance, as the clinician desires, but not both.

However the circumstance in question occurs when a clinician manual alters the PTV to remove the overlap with PRV. This is demonstrated in the next two figures. Figure 4a shows the dose distribution around the PTV $6000_{\text {unaltered }}$ (blue) which corresponds to the dotted line of Figure 3. Figure 4b shows the dose distribution around the PTV6000altered (red). You should carefully compare the colour wash distributions in these two images.

It is clear from the isodose distribution that the dose distribution is identical in the two plans. However, the PTV6000 from the two plans are different, as are the resultant DVHs (Figure 5). The red DVH (the PTV6000 altered) is "PERFECT". However the blue DVH (the PTV6000 unaltered)is "IMPERFECT" because the PTV coverage is 'cold', which is what prompts the alteration of PTV. Yet the dose distribution is identical! This is a classification problem. 


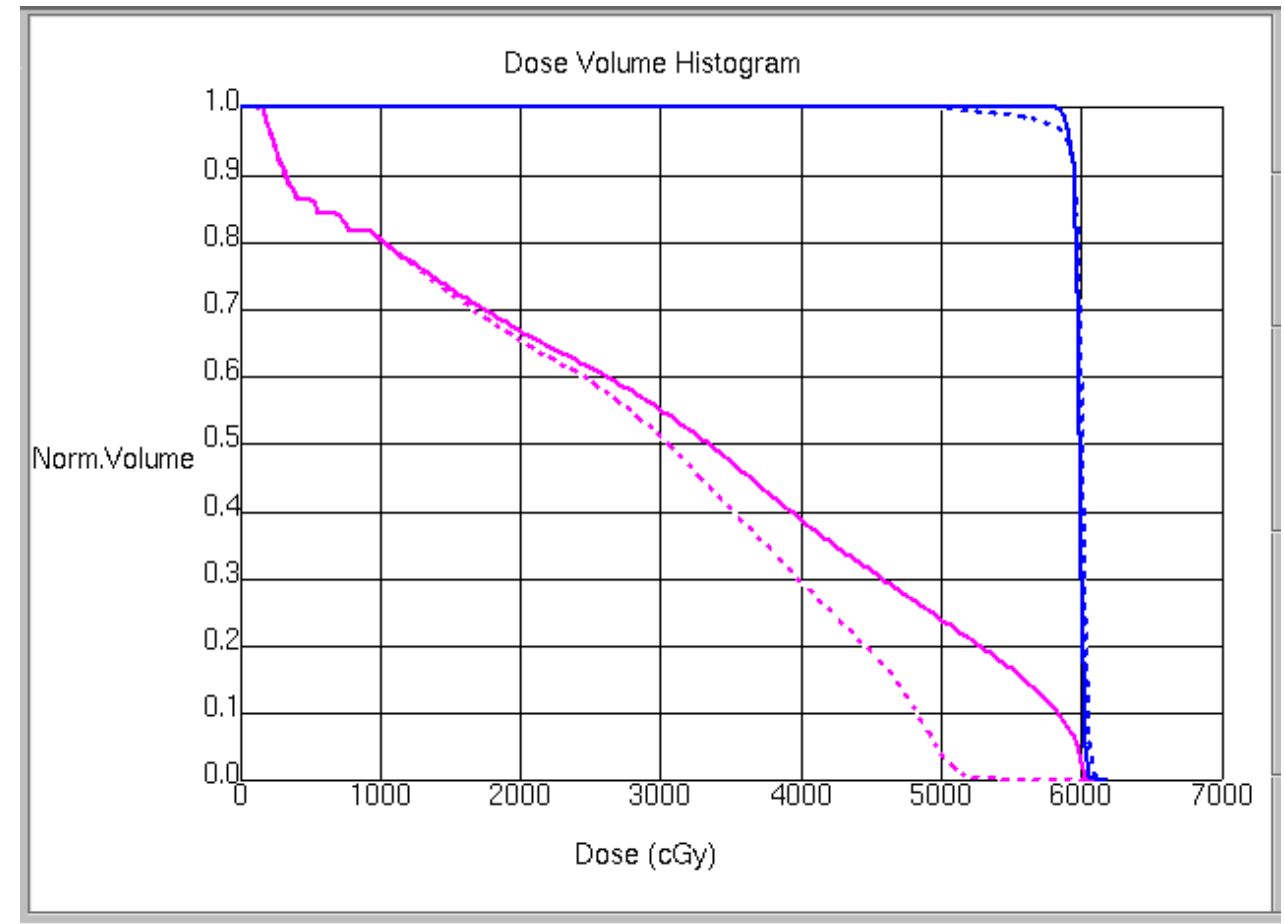

Plan 1: .....

Plan 2:

Figure 3: The DVH for Plan 1 [BRAINSTEM_PRV_4500 (purple) over PTV6000 (blue) - dotted lines] and Plan 2 [PTV6000 (blue) over BRAINSTEM_PRV_4500 (purple) - solid lines].

For a DVH to be 'truthful' it will represent clinical compromises. It is worth noting that, in making the choice to protect the BRAINSTEM, there is no issue of medical error or incompetence in the decision making since the isodose distribution is identical. The desire to prepare 'perfect' plans is perplexing because it hides reasonable decision-making.

\subsection{Is it helpful?}

The final question relates to the Informatics of Planning and the embedding of clinical decision making within the logical process of planning.

Informatics deals with data, information and its inherent structure which we describe as knowledge. When standardised nomenclature and ICRU-compliant OAR/GTV/CTV/PTV delineation is used, patterns of clinical decision making are embedded. This knowledge is contained in the names stored in the electronic files and database entries generated in clinical practice which can be accessed for automated analysis, i.e., human eyes 


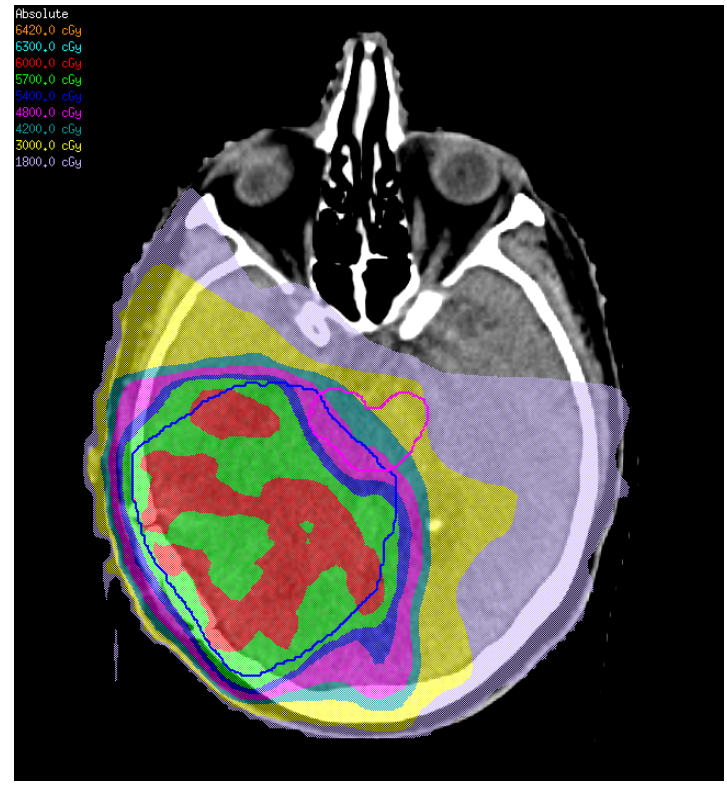

Figure 4a The $P T V 6000_{\text {unaltered is unchanged and }}$ overlaps BRAINSTEM_PRV_4500

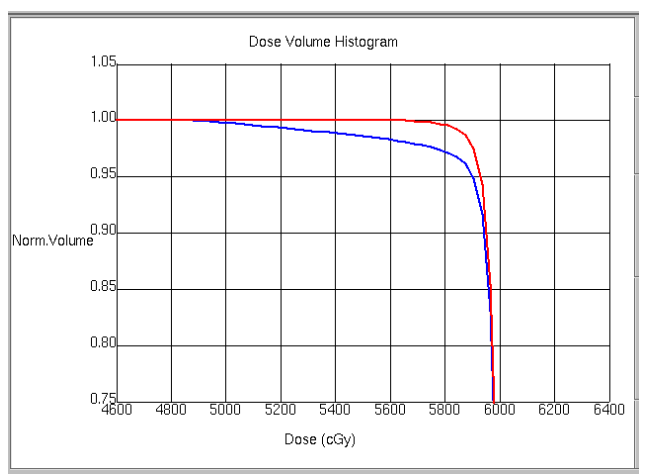

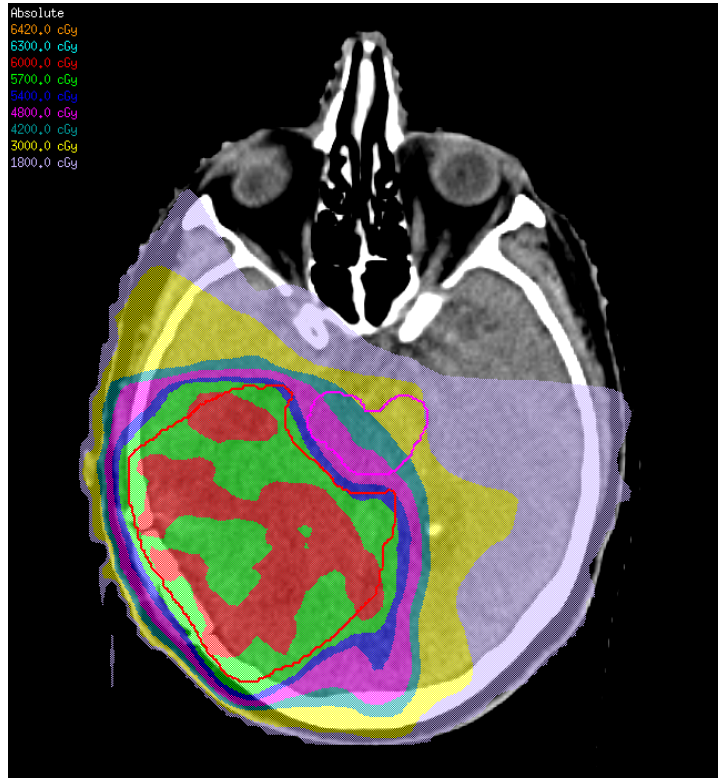

Figure 4b A PTV $6000_{\text {altered }}$ has been constructed to deliberately exclude BRAINSTEM_PRV_4500

Figure 5: The DVH for PTV6000altered

(red) and PTV6000 unaltered (blue)

don't have to see the contents of the files.

The ability to identify explicit decision making allows for the identification of better decision making in situations where oncologists don't know the answer. As already demonstrated, the issue is not where the isodose lines lie over the anatomy, but rather the ramifications of the decision. This article clearly demonstrates that if an oncologist wishes to respect cord tolerance by altering the PTV, it produces a different DVHs despite exactly the same isodose lines. This in essence moves the OAR away from the PTV.

On the one hand there is the 'IMPERFECT PLAN, PERFECT ANALYSIS' plan where the PTV and OAR are left unchanged. This requires no work from the oncologist, but asks for a risk-adjusted clinical planning priority that says "maximum OAR dose of 45Gy, minimum PTV dose of 45Gy". In this case the clinical decision making is obvious from the DVH because the two volumes can be shown to be overlapping with a dose that matches the OAR constraint. 
On the other hand there is the 'PERFECT PLAN, IMPERFECT ANALYSIS' plan where manually shaping separates the PTV from OAR. The perfect DVH obscures the clinical decision making because the perfect DVH imputes no decision making is required because of the spatial separation of the structures. This imputation is clearly false as the constructed structures do overlap. This circumstance embeds the conundrum of an OAR, which by vision that cannot be changed, and a PTV, which by definition cannot be altered. In this case the clinical decision making is not obvious from the DVH because the DVH is constructed from a PTV/OAR pair which are artificially non-overlapping.

An investigation to electronically assess the outcome of decision making to prioritize the PTV coverage or a specific OAR tolerance would access a PACS repository to access DICOM-RT files to see where the PTV coverage was acceptable but OAR coverage was excessive, or where OAR coverage was acceptable, but PTV coverage was deficient. Cases where PTV and OAR coverage are both acceptable would be ignored. In the selected cases, the outcome measures of local control and side effect occurrence would be correlated. The conclusion would be a measure of the consequences of each approach. Cases with an altered PTV would be missing from the analysis.

Irrespective of whether the control or complications are recorded electronically, when the PTV is altered, the clinical question of the consequences of respecting OAR tolerance or PTV dose cannot be answered because the overlap cannot be identified from DVH or DICOM-RT file, and because. And what cannot be detected cannot be correlated and analysed.

\section{Conclusion}

The practice of manual PTV alteration is a breach of the ICRU50/62/83 protocols defining what the PTV is and how it is derived. The decision of an oncologist cannot change the position of an anatomical structure. The decision of an oncologist cannot change the movement of a volume. The oncologist can only nominate CTV to PTV margins that are symmetrical, or occasionally anisotropic. The definitions of these terms are such that manual alteration of the properly generated PTV prevents the altered volume from being called a "PTV".

Given that the current DICOM-RT datasets do not include any clinical documentation describing the clinical decision making employed to construct a plan, only way that clinical decision making can be imputed is to utilise the defined terms accurately. Thus altering a PTV constructed according to the published ICRU definition is plainly misleading and is also not helpful in generating new knowledge using automated methods which are reliant on predictable contouring of OARs and construction of PTVs. 


\section{References}

[1] P. DeLuca, D Jones, R. Gahbauer, G. Whitmore, and A. Wambersie. ICRU The International Commission on Radiation Units and Measurements Report No. 83: Prescribing, Recording, and Reporting Photon-Beam IMRT. Journal of the ICRU, 10(1):1-106, April 2010.

[2] T. Landberg, J. Chavaudra, and J. Dobbs. ICRU The International Commission on Radiation Units and Measurements Report No. 50: Prescribing, Recording, and Reporting Photon Beam Therapy. Technical report, International Commission on Radiation Units and Measurements, 1993.

[3] A. Wambersie and T. Landberg. ICRU The International Commission on Radiation Units and Measurements Report No. 62: Prescribing, Recording, and Reporting Photon Beam Therapy (Supplement to ICRU report 50). Technical report, International Commission on Radiation Units and Measurements, 1999. 